\title{
Surgical management of vitreoretinal diseases in Bhutan: A 3-year national study
}

\author{
Bhim B Rai ${ }^{1,3 *}$, Michael G Morley², Pema Zangmo ${ }^{3}$, Thukten Tshering ${ }^{3}$, Abi N. Khatiwara ${ }^{3}$, Paul S Bernstein ${ }^{4}$ and Ted Maddess $^{1}$ \\ ${ }^{1}$ John Curtin School of Medical Research, Australian National University, Canberra, ACT, Australia \\ ${ }^{2}$ Ophthalmic Consultants of Boston, Harvard Medical School, Boston, MA, USA \\ ${ }^{3}$ Department of Ophthalmology, JDW National Referral Hospital, Thimphu, Bhutan \\ ${ }^{4}$ Moran Eye Centre, University of Utah School of Medicine, Salt Lake City, Utah, USA
}

\begin{abstract}
Purpose: To describe the pattern of vitreoretinal surgeries performed in Bhutan. This first national study informs policy making and resource development.

Methods: We reviewed the surgical registers of the three hospitals in Bhutan providing VR operations or interventional procedures for VR diseases over three years. Patient demography, indications and types of vitreoretinal surgeries were logged and quantified. Comparisons of the expected and observed frequency used Chisquared tests.

Results: A total of 214 patients received vitreoretinal surgeries, 36 paediatric cases received examination under anaesthesia for diagnostic confirmation followed by vitreoretinal surgery or retinal laser. Additionally, 381 cases received intravitreal anti-vascular endothelial growth factor injections in the operating theatres as mandated in Bhutan. The mean age was $56.2 \pm 22.3$ years. The majority, 375 cases $(59.4 \%)$ were males $(p<0.001)$. The common indications for VR surgery were retinal detachment (45.1\%), vitreous haemorrhage (13.6\%), and macular hole (7.7\%). Retinal detachment surgery was the most common surgery performed, followed by silicon oil removal with cataract surgery. The common indications for anti-vascular endothelial growth factor injection were neovascular age-related macular degeneration (168 cases, 44.12\%), retinal vein occlusion (132 cases, 34.6\%), and diabetic macular oedema and retinopathy (50 cases, $13.1 \%$ ).
\end{abstract}

Conclusion: Performing high-tech vitreoretinal surgeries in a resource-limited setting is challenging. With an ever-increasing load of vitreoretinal diseases such as retinal detachment, age-related macular degeneration and macular hole, and the complications of systemic diseases such as retinal vein occlusion, vitreous haemorrhage, and diabetic macular oedema and retinopathy, there is a need to improve vitreoretinal services in Bhutan.

\section{Introduction}

The origin of vitreoretinal surgery dates back to the early 1970 s, when Machemer first introduced pars plana vitrectomy (PPV) using a 17 Gauge $(\mathrm{G})$ cutter $[1,2]$. The cutter size was improved 20G by O'Mally and Heintz [3], 23G [4], 25G [5,6] and now 27G [7] for sutureless micro-incision vitrectomy. The invention of chandelier endoillumination further enhanced vitrectomy precision [8]. Sterile air was the first tamponade agent used by Ohm in 1911, but it was only in 1938 when Rosengren successfully treated retinal detachment (RD) with diathermy and air tamponade and coined the term tamponade [9]. Now we have newer tamponade agents such as silicon oil, sulphur hexafluoride (SF6), perfluoroethane (C2F6), and perfluoropropane (C3F8) [10]. Introduction of the wide-angle viewing systems was an important milestone: giving a panoramic view of the retina, essential for meticulous and successful VR surgery [11]. Application of these modern techniques is a relatively new in Bhutan.

Bhutan's first and only VR surgeon (BBR) completed his Fellowship in 2011 through the Himalayan Cataract Project (HCP) Scholarship program. With additional support from the HCP VR sub-speciality Clinic (VRSC) was established in 2012 at the Jigme Dorji Wangcheck National Referral Hospital (JDWNRH), Thimphu, Bhutan. The concept of right referrals was the cornerstone for success of the planned national VR services, therefore he established two extension VR clinics in the Eastern and Central Regional Referral Hospitals (RRHs), which were visited by his VR-team periodically. Additionally, he trained the optometrists and ophthalmic technicians posted in the feeder District Hospitals and Basic Health Units in remote areas through nationwide workshops on screening for diabetic eye diseases and other VR disorders. Another hurdle was to convince doctors treating systemic diseases affecting the eyes, such as diabetes and hypertension, to refer their potential VR patients to local optometrists and ophthalmic technicians for screening and making right referrals to the new national VR service. It is also critical to understand that proliferative diabetic retinopathy has concurrent involvement of other organs such as kidney associated with glycosuria and albuminuria [12]. With right referrals, health advocacy, and multidisciplinary coordination a successful national VRSC was established.

The authors have published articles on the pattern of VR diseases in Bhutan [13] and Nepal [14]. The current work is a complementary

*Correspondence to: Bhim Bahadur Rai, John Curtin School of Medical Research, Australian National University, Canberra, ACT 2601, Australia, E-mail: bhim.rai@anu.edu.au

Key words: vitreoretinal surgery, retinal surgery in Bhutan, retinal detachment, vitreous haemorrhage, anti-VEGF, macular hole

Received: July 05, 2020; Accepted: September 02, 2020; Published: September 09,2020 
study reporting on the pattern of VR surgeries and other VR procedures performed in Bhutan over study period. Since this is the first study on VR surgeries in Bhutan the results have impact for policy making and management of VR diseases in Bhutan and other developing countries. It also provides a baseline for future studies.

\section{Materials and methods}

\section{Participants}

All the patients who received VR operations or interventional procedures for VR diseases in the OT at the JDWNRH and Eastern and Central RRHs over three years, which extended from 01 May 2013 to 30 April 2016, were included in this study.

\section{Setting}

The study was conducted at the VRSC, Ophthalmology Department, JDWNRH, Thimphu, Bhutan, and includes the patients managed by the same VR surgeon in the Eastern and Central RRHs. The JDWNRH is the apex national referral hospital in the country. All the VR patients requiring surgery and laser therapy across the country are referred there for management, except when the VR-team visited the Eastern and Central RRHs. JDWNRH also serves as the main clinical centre for the postgraduate medical students, interns, and optometric and paramedic students.

\section{Study design and ethics}

This was a retrospective study, approved by the Research Ethics Board of Health (REBH), Ministry of Health, Royal Government of Bhutan, Thimphu, Bhutan. This study adhered to the principles of the Declaration of Helsinki. Consent was waived by REBH because this retrospective study collected only de-identified data.

\section{Clinical examination and data collection}

The details of medical history taking, clinical examinations, equipment and diagnostic procedures are given in a previous publication [13]. The surgical registers maintained in the VR OT were reviewed for the study period. The data collected included demographic information such as age and gender, indications for VR surgery, and types of surgical procedures performed.

In case of children, the diagnoses were sometimes confirmed by examination under anaesthesia (EUA), following a proper preanaesthetic check-up (PAC). If indicated, other procedures such as retinal laser in cases of retinopathy of prematurity (ROP) were delivered in the same sitting. However, if major surgical procedure was indicated a subsequent procedure was planned.

In the VR surgery the OT Constellation Vision System vitrector (Alcon Company, Texas, USA) was used. A green laser system (OcuLight Green, IRIDEX, Germany) was used for endolaser (EL) and indirect ophthalmoscopic delivery laser procedures in the VR OT.

\section{Quantification and statistical analysis}

The data were analysed using MATLAB (2016b, The MathWorks, Natick, MA), including tabulating and checking frequencies of cases and creating figures and tables. Comparisons of the expected and observed frequency of gender used Chi-squared tests. Further details are given in an allied review of retinal disease in Bhutan [13] (Figure 1).

\section{Results}

\section{Demography}

A total of 214 patients received VR surgeries, and 36 paediatric cases received EUA followed by VR surgery or retinal laser. Additionally, 381 patients received anti-VEGF injections in the OT over the study period. The mean age at presentation was $56.2 \pm 22.3$. The majority (374) were males, and 256 cases females $(40.6 \%)(\mathrm{p}<0.001)$. Figure 1 shows the distributions of the ages of the patients by gender (Table 1 ).

\section{VR surgeries}

$\mathrm{RD}$ was the commonest VR disease indicated for VR surgery, accounting for 55 cases (23.4\%). This was followed by cases who had already received $\mathrm{RD}$ surgery but needed secondary surgeries such as silicon oil (SO) removal following successful surgery, or as part of procedure for alleviating complications secondary to $\mathrm{RD}$ surgery. Thus, status post RD surgery as an indication was seen in 51 cases $(21.7 \%)$, and in total 106 cases (45.1\%) were operated for RD as an indication. Vitreous haemorrhage $(\mathrm{VH})$, secondary to trauma, diabetic retinopathy (DR), retinal vein occlusion (RVO), and other retinal vasculitis, was seen in 32 cases (13.6\%). Other indications were full-thickness macular hole $(\mathrm{MH})$ in 18 cases $(7.7 \%)$, dislocated cataract in 16 cases $(6.8 \%)$, vitreomacular traction (VMT) or fibrovascular membrane (FVM) in 11 cases $(4.7 \%)$, dislocated and decentered intraocular lens (IOL) in 9 cases (3.8\%), endophthalmitis in 9 cases (3.8\%), congenital cataract in 8 cases (3.4\%), ROP in 8 cases (3.4\%), ectopia lentis such as Marfan's syndrome in 5 cases $(2.1 \%)$. In the subgroup of 9 cases of endophthalmitis 5 were traumatic, 2 cases following penetrating keratoplasty, 1 case after cataract operation with IOL implantation and 1 case of endogenous endophthalmitis. There were four cases of retained intraocular foreign body (RIOFB). One of them also had rhegmatogenous RD secondary to the RIOFB and had successful RIOFB removal and RD surgery. Other indications are shown in Table 2.

RD surgery was the most common VR surgery performed in 52 cases (22.9\%). The procedures involved PPV, fluid-air exchange (FAE), $\mathrm{EL}$ and injection of SO for internal tamponade (5 cases received $\mathrm{C} 3 \mathrm{~F} 8$ ). Scleral band buckling (SBB) and membrane peeling were performed

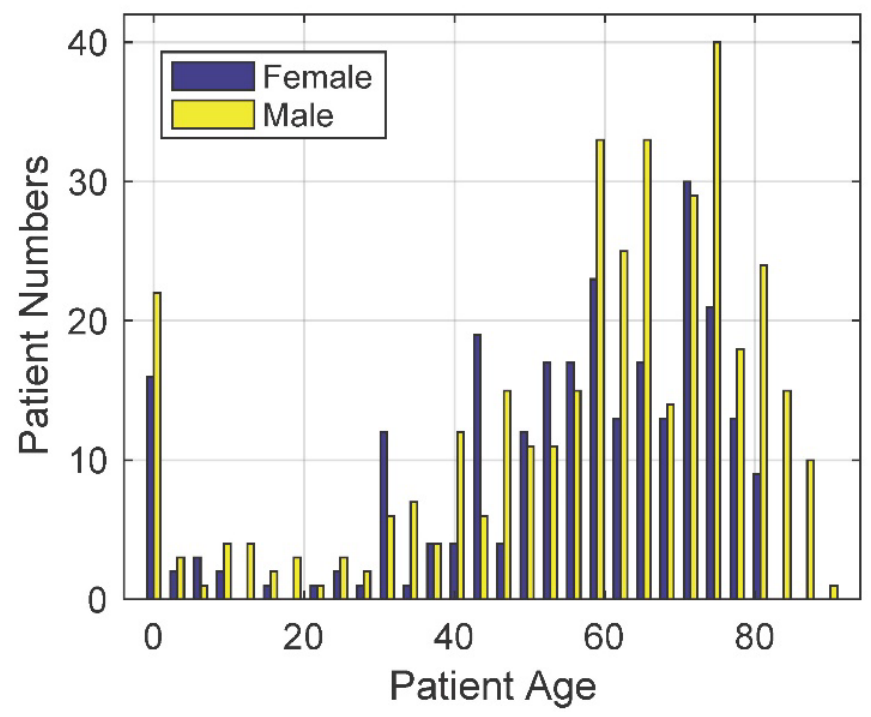

Figure 1. Age distributions of the patients in the study by gender 
in 27 and 25 of the RD cases respectively. Silicon oil removal (SOR) combined with small incision cataract surgery (SICS) and intraocular lens (IOL) implantation was the second most common VR surgery performed in 45 cases (19.8\%). PPV combined with lensectomy and IOL implantation was done in 28 cases (12.3\%). In these cases, manual SICS was done additionally if rigid IOL implantation was planned. PPV combined with MP and EL was done 21 cases (9.3\%). MH surgery was done in 18 cases $(7.9 \%)$. This procedure involved PPV with internal limiting membrane (ILM) peeling with intravitreal injection of SF6 gas. PPV with removal of dislocated cataract or IOL was done in 16 cases $(7.0 \%)$. Dislocated cataracts were mostly removed using the fragmatome procedure, a functional extension of the Constellation Vision System. Rigid IOLs were removed through the sclero-corneal tunnel incision. PPV (core vitrectomy) with intravitreal injections of antibiotics, with or without steroids, was done in 12 cases $(5.3 \%)$. These were the cases of postoperative and traumatic endophthalmitis. Along with vitreous biopsy procedure for culture, and sensitivity tests for microbiology were also done. PPV with IOL reposition or exchange was done in 9 cases $(4.0 \%)$. These cases had fully dislocated IOL into the vitreous cavity, or symptomatic subluxated or decentered IOLs affecting vision. PPV with other procedures was done in another 9 cases $(4.0 \%)$. Retinal laser under general anaesthesia (EUA) was done in 8 cases $(3.5 \%)$ of ROP. The details of the surgeries are summarised in Table 3. Four cases (1.8\%) of retained intraocular foreign body (RIOFB) were operated. PPV was done and RIOFBs were removed manually with intraocular forceps or magnet through the extended PPV port, or corneo-scleral tunnel incision. One of the four RIOFB cases also had associated $\mathrm{RD}$, which was managed with proper $\mathrm{RD}$ surgery procedure following removal of the RIOFB.

Table 1. Demographic characteristics of patients by basic surgery category. p-values indicate the significance of the difference in gender frequencies compared to $50 \%$.

\begin{tabular}{|c|c|c|c|c|c|}
\hline & N & Age & Males & Females & $p$ \\
\hline $\begin{array}{c}\text { Vitreo-retinal } \\
\text { (VR) surgery }\end{array}$ & 214 & $49.5 \pm 21.0$ & 122 & 92 & 0.147 \\
\hline $\begin{array}{c}\text { EUA* } \pm \text { VR } \\
\text { surgery /retinal } \\
\text { laser }\end{array}$ & 36 & $1.21 \pm 2.27$ & 22 & 14 & 0.346 \\
\hline $\begin{array}{c}\text { Injection anti- } \\
\text { VEGF** }\end{array}$ & 381 & $65.2 \pm 13.4$ & 230 & 151 & 0.004 \\
\hline
\end{tabular}

*EUA $=$ Examination under anaesthesia; $\mathrm{VEGF}^{* *}=$ Vascular endothelial growth factor.

Table 2. Indications for Vitreo-retinal surgery

\begin{tabular}{|c|c|c|}
\hline Indications & Number of cases & Percentage \\
\hline Retinal Detachment (RD) & 55 & 23.4 \\
\hline Status post RD Surgery & 51 & 21.7 \\
\hline Vitreous haemorrhage* & 32 & 13.6 \\
\hline Full-thickness macular hole & 18 & 7.7 \\
\hline Dislocated Cataract & 16 & 6.8 \\
\hline Vitreo-macular traction + Fibrovascular & 11 & 4.7 \\
\hline membrane & 9 & 3.8 \\
\hline Dislocated and decentered Intraocular lens & 9 & 3.8 \\
\hline Post-operative endophthalmitis & 8 & 3.4 \\
\hline Congenital Cataract & 8 & 3.4 \\
\hline Retinopathy of prematurity & 5 & 2.1 \\
\hline Traumatic Endophthalmitis & 5 & 2.1 \\
\hline Ectopia Lentis & 4 & 1.7 \\
\hline Retained intraocular foreign body & 4 & 1.7 \\
\hline Others** & 235 & 100.0 \\
\hline Total & & \\
\hline
\end{tabular}

*VH=Traumatic, Diabetic retinopathy, RVO, Retinal vasculitis

**Others=Epiretinal membrane, vitreous opacities with pupillary and anterior hyaloid membranes, masquerades syndrome
Table 3. Types of Vitreo-retinal surgery

\begin{tabular}{|c|c|c|}
\hline Type & Number of cases & Percentage \\
\hline $\begin{array}{l}\text { RD Surgery }( \pm \mathrm{SBB}+\mathrm{PPV} \pm \mathrm{MP} \\
+\mathrm{FAE}+\mathrm{EL}+\mathrm{SOI} \text { or } \mathrm{C} 3 \mathrm{F8})\end{array}$ & 52 & 22.9 \\
\hline SOR+SICS+IOL & 45 & 19.8 \\
\hline PPV+Lensectomy/SICS+IOL & 28 & 12.3 \\
\hline $\mathbf{P P V}+\mathbf{M P}+\mathbf{E L}$ & 21 & 9.3 \\
\hline $\begin{array}{c}\text { Macular Hole Surgery (PPV+ILM } \\
\text { peeling+SF6) }\end{array}$ & 18 & 7.9 \\
\hline PPV+Removal of dislocated cataract/IOL & 16 & 7.0 \\
\hline PPV+Intra-vitreal injection of antibiotics & 12 & 5.3 \\
\hline PPV+IOL reposition/exchange & 9 & 4.0 \\
\hline PPV+other procedures & 9 & 4.0 \\
\hline Retinal laser for ROP & 8 & 3.5 \\
\hline PPV+MP+SICS+IOL+EL+SOI & 5 & 2.2 \\
\hline PPV+RIOFB removal & 4 & 1.8 \\
\hline Total & 227 & 100.0 \\
\hline
\end{tabular}

Eleven cases excluded due to insufficient data. C3F8: perfluoropropane; EL: endolaser; FAE: Fluid-air exchange; ILM: internal limiting membrane; IOL: intraocular lens; MP: membrane peeling; PPV: pars plana vitrectomy; RIOFB: retained intraocular foreign body; ROP: retinopathy of prematurity; SBB: scleral band buckling; SF6: sulphur hexafluoride; SICS: small incision cataract surgery; SOI: silicon oil injection; SOR: silicon oil removal.

\section{Examinations under anaesthesia}

A total of 36 cases, mainly paediatric, received EUA following PCA. Within this subgroup, the majority were cases of ROP (24 cases). Eight of the 24 ROP cases required further surgical interventions in the form of retinal lasers, or RD surgery. The option of administering anti-VEGF was contemplated over laser therapy, but due to limited resources and limited funding laser therapy was commonly performed. There were 2 cases each of tractional RD and retinoblastoma. The retinoblastoma cases were referred to an advanced cancer centre in India. There was one case each of Coat's disease, retinitis pigmentosa (RP), traumatic retinal haemorrhage, congenital glaucoma, and Marfan's syndrome. Three cases undergoing EUA had normal retinas. The types of cases receiving EUA are shown in Supplementary Table S1.

Indications of intravitreal anti-VEGF injections among 381 patients who received it in the OT (as mandated in Bhutan) during the study period are shown in Table S2. Neovascular AMD (nAMD) was the most common indication for the injection (168 cases, 44.2\%). Others included RVO (132 cases, 34.6\%), DMO/DR (50 cases, 13.1\%), myopic choroidal neovascular membrane (mCNVM) (11 cases, $0.03 \%)$, and polypoidal choroidal vasculopathy (PCV) (6 cases, $0.02 \%)$. Within the RVO subgroup, branch retinal vein occlusion (BRVO) was the most common subgroup with 70 cases $(53.0 \%$ of RVO cases), followed by central retinal vein occlusion (CRVO) in 55 cases (41.7\%) and hemiretinal BRVO (HRBRVO) in 7 cases 5.3\%).

\section{Discussion}

Our study on the pattern and presentation of VR diseases in Bhutan found that majority of patients were males [13]. This could explain why the majority of patients in this study receiving surgical management were also males. A meta-analysis of eye surgeries in developing countries reported that females receive fewer surgical services than males [15].

Here RD was the most common indication for VR surgery even though RD was reported by our prevalence study as being the seventh most common VR disease in Bhutan [13]. What caused this? The VRSC was established in Bhutan in early 2012 when the first national VR surgeon (BBR) joined the National Health Service. Before that VR 
cases, including RD, were referred to India or Nepal. Most patients were reluctant to leave Bhutan for surgery, and consequently received no treatment, or employed traditional medicine sometimes resulting in irreversible loss of vision and even the loss of eyes. Therefore, after establishment of the VR service the number of RD cases was relatively large, and RD surgery was the most common VR surgery performed. That being said other relevant studies have reported higher prevalence of RD in Nepal [14], and more among Chinese than Indians in Singapore [16].

Before the national VR service Bhutanese patients returning home after surgery often had limited understanding of their condition and their post-operative care, due to language and cultural differences. Some of them had SO in their eyes for years, which converted into complications like secondary cataract, glaucoma, corneal decompensation, and failure of retinal attachment. Thus, SOR combined with SICS and IOL implantation was the second most common surgery performed over the study period. BBR preferred to use SO over expansible gases as the tamponade to manage $\mathrm{RD}$ because it is more likely to: produce reattachment, better postoperative visual acuity, and fewer postoperative complications $[17,18]$. Although the optimal timing for the SOR remains debatable, 3 to 6 months are commonly recommend for sustained retinal attachment $[19,20]$. Some retinal experts suggest SOR should be performed no longer than 6 months after its injection, and the best timing being 2 to 3 months post-injection [21], possibly avoiding complications such as keratopathy, glaucoma and visual loss [22].

VH secondary to DR, RVO, trauma and other retinal vasculitis, was the second commonest indication for VR surgery because of the high prevalence of HTR, DR, RVO and retinal vasculitis in Bhutan [13], as in nearby Nepal [14]. This demanded PPV to clear obstructive VH, with or without EL, to be performed commonly both for therapeutic and diagnostic purposes. For the same reason surgical procedures for FVM, VMT and tractional RD were also commonly performed because many of these were caused by highly prevalent DR, HTR and RVO [13]. There were eighteen cases of full-thickness $\mathrm{MH}$ received surgery during the study period although there were 76 eyes diagnosed with the condition [13]. This low surgical rate might be due to poor functional outcomes despite good success of $\mathrm{MH}$ surgery in general [23,24], which was explained to the patients and their party during counselling sessions before surgery. Another reason could be extended waiting times for surgery due to restricted availability of consumables required for $\mathrm{MH}$.

Trauma was the fifth most common cause of injury requiring VR surgery in Bhutan [13], which resulted in cases of traumatic endophthalmitis and dislocated or subluxated cataract or IOL. Therefore, PPV with intravitreal injections of antibiotics with and without steroids to manage endophthalmitis, and PPV for retrieval, explant, reposition and exchange of IOL were performed commonly. Similarly, PPV for extraction of dislocated and subluxated cataract were commonly performed. Traumatic cases with associated RIOFB were managed with PPV and removal of foreign body with intraocular forceps through extended PPV port or corneoscleral tunnel. One such case developed RD secondary to RIOFB, which was managed with PPV with RIOFB removal combined with RD surgery. The case developed secondary cataract requiring SICS with IOL implantation successfully [25].

Although the number of ROP cases needing intervention was small (8) we feel this condition needs more attention. Firstly, the incidence of ROP is increasing world-wide [26-28], due to the increasing incidence of infants born at 24 weeks or earlier [26]. Increased oxygen use has improved survival rates and reduced cerebral palsy, but has increased the incidence of ROP [27]. The number of life-years affected by ROP is large [29]. Thus, it was estimated in 1996 that the global financial cost of childhood blindness in terms of lost earning capacity was between US $\$ 6$ trillion and $\$ 27$ trillion, surpassing the cost of adult blindness [30]. The generational cost-savings for society is approximately $\$ 516$ million [31]. Finally, ROP management falls in the grey area between the VR specialist and the paediatric ophthalmologist, so there is a risk to proper follow up. Strong partnering between paediatric ophthalmologists, VR specialists, parents and neonatologists is therefore recommended, along with proper follow up mechanisms.

nAMD was the most common indication for anti-VEGF injection because AMD is the fourth most common VR disease in Bhutan [13]. Considering HTR and diabetes as risk factors for RVO [32], and both being common among the Bhutanese population, RVO cases received the second highest number of anti-VEGF injections. Although DR and DMO were the third most common VR diseases in Bhutan, and second most common in a related Nepal study [14], only a small proportion received anti-VEGF injection. Majority of such cases were managed with retinal laser. Only a small percentage of anti-VEGF injections was given to $\mathrm{mCNVM}$ cases because the prevalence of $\mathrm{mCNVM}$ among the myopes in Bhutan was only $0.4 \%$ [33]. Other studies have reported that mCNVM occurs in approximately $5-10 \%$ of highly myopic patients, and $0.04-0.05 \%$ of the general population $[34,35]$.

This study is limited by the number of patients receiving VR surgery. Considering the national population of less than a million [36] the case number reported here is considerable. Unfortunately, we do not have data on visual acuity or outcomes of the surgery. Eleven operative cases were excluded due to incomplete data.

\section{Conclusions}

While VR services in Bhutan have advanced greatly in the last decade there is room for improvement, especially for common VR diseases such as AMD, DR, DMO, RVO, and myopic degenerations resulting in vision threatening atrophic retinal hole, $\mathrm{mCNVM}$, and rhegmatogenous RD. Unfortunately, VR diseases secondary to systemic diseases such as hypertension and diabetes remain common [13]. There is an urgent need for collaboration between ophthalmologists, physicians, nutritionists, the patients and public to ameliorate these diseases. Yet another major priority is tackling ROP. A strong partnership between paediatric ophthalmologists, VR specialists, neonatologists, and parents is recommended.

\section{Declarations}

\section{Funding}

The study was supported by the John Curtin School of Medical Research at the Australian National University.

\section{Conflict of interest}

Author BBR declares that he has no conflict of interest. Author MGM declares that he has no conflict of interest. Author PZ declares that she has no conflict of interest. Author TT declares that he has no conflict of interest. Author ANK declares that he has no conflict of interest. Author PSB declares that he has no conflict of interest. Author TM declares that he has no conflict of interest.

\section{Availability of data and materials}

The data and materials are available with the first author BBR. 


\section{Ethical approval and standards}

The study has been approved by the Research Ethics Board of Health (REBH), Ministry of Health, Royal Government of Bhutan, Thimphu, Bhutan, dated 06/12/2016 through approval number REBH/ Approval/2016/083. All procedures performed in studies involving human participants were in accordance with the ethical standards of the REBH and in line with the principles of the Declaration of Helsinki.

\section{Authors' contributions}

BBR conceived of the study, collected data, did analysis and wrote the manuscript. MGM guided BBR and reviewed the manuscript. $\mathrm{PZ}$ collected data and reviewed the manuscript. TT collected data and reviewed the manuscript. ANK collected data and reviewed the manuscript. PSB guided BBR and reviewed the manuscript. TM helped with the analysis, reviewed the manuscript, and guided BBR closely. All authors read, reviewed, and approved the final manuscript.

\section{References}

1. Machemer R (1972) A new concept for vitreous surgery. 2. Surgical technique and complications. Am J Ophthalmol 74: 1022-1033. [Crossref]

2. Machemer R, Norton EW (1972) A new concept for vitreous surgery. 3. Indications and results. Am J Ophthalmol 74: 1034-1056. [Crossref]

3. O'Mally C, Heintz RM (1975) Vitrectomy with an alternative instrument system. Ann of Ophthalmol 7: 585-588. [Crossref]

4. Eckardt C (2005) Transconjunctival sutureless 23-gauge vitrectomy. Retina 25: 208211. [Crossref]

5. de Juan E, Hickingbotham D (1990) Refinements in microinstrumentation for vitreous surgery. Am J Ophthalmol 109: 218-220. [Crossref]

6. Fujii GY, de Juan E, Humayun MS, Pieramici DJ, Chang TS, et al. (2002) A new 25 -gauge instrument system for transconjunctival sutureless vitrectomy surgery. Ophthalmology 109: 1807-1812. [Crossref]

7. Oshima Y, Wakabayashi T, Sato T, Ohji M, Tano Y (2010)A 27-gauge instrument system for transconjunctival sutureless microincision vitrectomy surgery. Ophthalmology 117 : 93-102.e102. [Crossref]

8. Oshima Y, Awh CC, Tano Y (2007) Self-retaining 27-gauge transconjunctival chandelier endoillumination for panoramic viewing during vitreous surgery. Am $\mathrm{J}$ of Ophthalmol 143: 166-167.e161. [Crossref]

9. Rosengren B (1938) Results of treatment of detachment of the retina with diathermy and injection of air into the vitreous. Acta Ophthalmol 16: 573-579.

10. Vaziri K, Schwartz SG, Kishor KS, Flynn HW Jr. (2016) Tamponade in the surgical management of retinal detachment. Clin Ophthalmol 10: 471-476.

11. de Oliveira PR, Berger AR, Chow DR (2016) Vitreoretinal instruments: vitrectomy cutters, endoillumination and wide-angle viewing systems. Intl J Retina Vitreous 2: 28.

12. Thapa R, Bajimaya S, Sharma S, Rai BB, Paudyal G (2014) Systemic association of newly diagnosed proliferative diabetic retinopathy among type 2 diabetes patients presented at a tertiary eye hospital of Nepal. Nepal J Ophthalmol 7: 26-32. [Crossref]

13. Rai BB, Morley MG, Bernstein PS, Maddess T (2020) Pattern of vitreo-retinal diseases at the national referral hospital in Bhutan: a retrospective, hospital-based study. $B M C$ Ophthalmol 20: 51. [Crossref]

14. Rai BB, Shrestha MK, Thapa R, Essex RW, Paudyal G, et al. (2019) Pattern and Presentation of Vitreo-retinal Diseases: an analysis of retrospective data at a tertiary eye-care centre in Nepal. Asia-Pacific J Ophthalmol 8: 481-488. [Crossref]

15. Lewallen S, Courtright P (2002) Gender and use of cataract surgical services in developing countries. World Health Organization. https://www.who.int/bulletin/ archives/80(4)300.pdf. Accessed November 52019
16. Wong TY, Tielsch JM, Schein OD (1999) Racial difference in the incidence of retinal detachment in Singapore. Arch Ophthalmol 117: 379-383.

17. Group TSS (1992) Vitrectomy with silicone oil or sulfur hexafluoride gas in eyes with severe proliferative vitreoretinopathy: results of a randomized clinical trial. Silicone Study Report 1. Arch Ophthalmol 110: 770-779. [Crossref]

18. Abrams GW, Azen SP, McCuen BW, Flynn HW Jr, Lai MY, et al. (1997) Vitrectomy with silicone oil or long-acting gas in eyes with severe proliferative vitreoretinopathy: results of additional and long-term follow-up. Silicone Study report 11. Arch Ophthalmol 115 (3):335-344. [Crossref]

19. Falkner CI, Binder S, Kruger A (2001) Outcome after silicone oil removal. $\mathrm{Br} J$ Ophthalmol 85: 1324-1327. [Crossref]

20. Casswell AG, Gregor ZJ (1987) Silicone oil removal. II. Operative and postoperative complications. Br J Ophthalmol 71: 898-902. [Crossref]

21. Jiang Y, Li X (1997) The best timing of silicone oil removal. Zhonghua Yan Ke Za Zhi 33: 39-41. [Crossref]

22. Hocaoglu M, Karacorlu M, Ersoz MG, Sayman Muslubas I, Arf S (2019) Vitrectomy with silicone oil tamponade for retinal detachment associated with giant retinal tears: Favourable outcomes without adjuvant scleral buckling. Acta Ophthalmol 97: E271-E276. [Crossref]

23. Zhao PP, Wang S, Liu N, Shu ZM, Zhao JS (2018) A review of surgical outcomes and advances for macular holes. J Ophthalmol 7389412.

24. Haritoglou C, Gass CA, Schaumberger M, Ehrt O, Gandorfer A, et al. (2001) Macular changes after peeling of the internal limiting membrane in macular hole surgery. $\mathrm{Am} \mathrm{J}$ Ophthalmol 132: 363-368. [Crossref]

25. Rai BB, Dorji S, Zangmo P, Tshering T (2016) Retained intraocular metallic foreign body causing retinal detachment. Bhutan Health $J$ 2: 48-52.

26. Isaza G, Donaldson L, Chaudhary V (2019) Increased incidence of retinopathy of prematurity and evolving treatment modalities at a Canadian tertiary centre. Canadian J of Ophthalmol 54: 269-274. [Crossref]

27. Holmstrom G, Tornqvist K, Al-Hawasi A, Nilsson A, Wallin A, et al. (2018) Increased frequency of retinopathy of prematurity over the last decade and significant regional differences. Acta Ophthalmol 96: 142-148. [Crossref]

28. Freitas AM, Morschbacher R, Thorell MR, Rhoden EL (2018) Incidence and risk factors for retinopathy of prematurity: a retrospective cohort study. Int J Retina Vitreous 4: $1-8$.

29. Rahi JS, Gilbert CE, Foster A, Minassian D (1999) Measuring the burden of childhood blindness. Br J Ophthalmol 83: 387-388. [Crossref]

30. Decarlo DK, McGwin G Jr, Bixler ML, Wallander J, Owsley C (2012) Impact of pediatric vision impairment on daily life: results of focus groups. Optom Vis Sci 89: 1409-1416. [Crossref]

31. Dave HB, Gordillo L, Yang Z, Zhang MS, Hubbard GB, 3rd, et al. (2012) The societal burden of blindness secondary to retinopathy of prematurity in Lima, Peru. Am J Ophthalmol 154: 750-755.

32. Kolar P (2014) Risk factors for central and branch retinal vein occlusion: a metaanalysis of published clinical data. J Ophthalmol 2014: 724780. [Crossref]

33. Rai BB, Ashby R, French A, Maddess T (2020) Rural-urban differences in Myopia prevalence among myopes presenting to Bhutanese retinal clinical services: a 3-year national study. Graef Arch Clin Exp Ophthalmol. [Crossref]

34. Avila MP, Weiter JJ, Jalkh AE, Trempe CL, Pruett RC, et al. (1984) Natural history of choroidal neovascularization in degenerative myopia. Ophthalmology 91: 1573-1581.

35. El Matri L, Chebil A, Kort F (2015) Current and emerging treatment options for myopic choroidal neovascularization. Clin Ophthalmol 9: 733-744. [Crossref]

36. Population and Housing Census of Bhutan 2017 (2017) National Report. http://www nsb.gov.bt/publication/files/PHCB2017_national.pdf. Accessed 2019 January

Copyright: (C2020 Rai BB. This is an open-access article distributed under the terms of the Creative Commons Attribution License, which permits unrestricted use, distribution, and reproduction in any medium, provided the original author and source are credited. 